\title{
Assessment: Botulinum neurotoxin for the treatment of movement disorders (an evidence-based review)
}

\author{
Report of the Therapeutics and Technology Assessment \\ Subcommittee of the American Academy of Neurology \\ [a
}

D.M. Simpson, MD

A. Blitzer, MD, DDS

A. Brashear, MD

C. Comella, MD

R. Dubinsky, MD, $\mathrm{MPH}$

M. Hallett, MD

J. Jankovic, MD

B. Karp, MD

C.L. Ludlow, PhD

J.M. Miyasaki, MD, MEd

M. Naumann, MD

Y. So, MD, PhD

Address correspondence and reprint requests to the American Academy of Neurology, 1080 Montreal Ave., St. Paul, MN 55116 guidelines@aan.com

Supplemental data at www.neurology.org

See pages 1691 and 1707

\section{ABSTRACT}

Objective: To perform an evidence-based review of the safety and efficacy of botulinum neurotoxin (BoNT) in the treatment of movement disorders.

Methods: A literature search was performed including MEDLINE and Current Contents for therapeutic articles relevant to BoNT and selected movement disorders. Authors reviewed, abstracted, and classified articles based on American Academy of Neurology criteria (Class I-IV).

Results: The highest quality literature available for the respective indications was as follows: blepharospasm (two Class II studies); hemifacial spasm (one Class II and one Class III study); cervical dystonia (seven Class I studies); focal upper extremity dystonia (one Class I and three Class II studies); focal lower extremity dystonia (one Class II study); laryngeal dystonia (one Class I study); motor tics (one Class II study); and upper extremity essential tremor (two Class II studies).

Recommendations: Botulinum neurotoxin should be offered as a treatment option for the treatment of cervical dystonia (Level A), may be offered for blepharospasm, focal upper extremity dystonia, adductor laryngeal dystonia, and upper extremity essential tremor (Level B), and may be considered for hemifacial spasm, focal lower limb dystonia, and motor tics (Level C). While clinicians' practice may suggest stronger recommendations in some of these indications, evidencebased conclusions are limited by the availability of data. Neurology ${ }^{\circledR}$ 2008;70:1699-1706

\section{GLOSSARY}

ABSD = abductor type of spasmodic dysphonia; ADSD = adductor type of spasmodic dysphonia; BoNT = botulinum neurotoxin; $C D=$ cervical dystonia, FDA = Food and Drug Administration.

INTRODUCTION Botulinum neurotoxin (BoNT) has emerged as an effective treatment for numerous movement disorders associated with muscle overactivity. Two companion articles provide reviews of the pharmacology and immunology of BoNT, and an eyidence-based review of its use in spasticity, ${ }^{1}$ autonomic disorders, and pain. ${ }^{2}$ This article evaluates the current knowledge and evidence of BoNT in selected movement disorders.

\section{DESCRIPTION OF THE ANALYTICAL PROCESS}

The literature search strategy, panel formation, and literature analytic process are described in the companion article on BoNT in the treatment of spasticity. ${ }^{1}$ Since the different preparations of BoNT have different potencies and durations of action, the serotype and brand of BoNT used in specific studies are provided in the evidence tables, but the text distinguishes their effects only when the data are sufficient to do so, or when referring to specific dosages.

Blepharospasm. Blepharospasm is a focal dystonia characterized by involuntary contraction of orbicularis oculi, causing involuntary closure of the

\footnotetext{
From the Department of Neurology (D.M.S.), Mount Sinai Medical Center, New York; Department of Otolaryngology (A. Blitzer), St. Lukes-Roosevelt Medical Center, New York, NY; Wake Forest University School of Medicine (A. Brashear), Winston-Salem, NC; Department of Neurology (C.C.), Rush University Medical Center, Chicago, IL; Department of Neurology (R.D.), University of Kansas, Kansas City; The National Institute of Neurological Disorders and Stroke (M.H., B.K., C.L.L.), Bethesda, MD; Department of Neurology (J.J.), Baylor College of Medicine, Houston, TX; Toronto Western Hospital (J.M.M.), Ontario, Canada; Department of Neurology (M.N.), Klinikum Augsburg, Germany; and Stanford University (Y.S.), CA.

Approved by the Therapeutics and Technology Assessment Subcommittee on March 31, 2007; by the Practice Committee on July 12, 2007; and by the AAN Board of Directors on January 30, 2008.

The Mission Statement, Conflict of Interest Statement, Subcommittee and Panel members, AAN classification of evidence, and Classification of recommendations are available as supplemental data on the Neurology ${ }^{\circledR}$ Web site at www.neurology.org.

Endorsed by the American Academy of Physical Medicine and Rehabilitation on March 14, 2008.

Disclosure: Author disclosures are provided at the end of the article.
} 
eyes. Prior to BoNT, there were no effective medical or surgical treatments for this disorder. Blepharospasm was one of the first studied indications for BoNT treatment. The open label observations were felt to be so dramatic that there have been only a few attempts to perform properly controlled clinical trials. In 1989, Botox ${ }^{\circledR}$ received Food and Drug Administration (FDA) approval for blepharospasm, including benign essential blepharospasm or VII nerve disorders, in patients 12 years and older. Two efficacy trials fulfilled criteria for Class II (table e-1 on the Neurology ${ }^{\circledR}$ Web site at www.neurology.org).

One Class II trial was a double-blind comparison of injecting BoNT-A into one eyelid and saline into the other. ${ }^{3}$ Six patients received BoNT-A (Botox ${ }^{\circledR}$ ) at a dose of 20 U/eye for the active treatment. Blinded rating of videotapes showed bilateral reduction in blepharospasm that was greater on the side injected with active toxin. The other Class II study was a doubleblind, prospective, crossover trial of 11 patients using BoNT-A (Botox ${ }^{\circledR}$ ) at 25 U/eye, supplementing up to $50 \mathrm{U} /$ eye 1 month later if needed. ${ }^{4}$ Assessment by physician observation and rating of videotapes, using the Fahn scale and by patient subjective rating, showed significant efficacy lasting a mean of 2.5 months. Adverse effects were generally mild (blurred wision, tearing, ptosis, and ecchymosis).

One Class II and one Class III study compared two different brands of BoNT-A $\left(\right.$ Botox $^{\circledR}$ and Dysport $\left.^{\circledR}\right)$. In the Class II study, there were 212 patients eyaluated in a crossover design using a 4:1 dose ratio of Dysport ${ }^{\circledR}$ to Botox ${ }^{\circledR}$. 5 The primary endpoint, duration of effect, was similar for the two products. The Class III study used a parallel design of 42 patients without blinded raters and also used a dose ratio of $4: 1 .^{6}$ Duration of action was again the primary endpoint, and this endpoint and others including number of booster doses needed, latency of effect, clinical efficacy, and adverse reactions were similar for the two products. A Class I study compared Xeomin ${ }^{\circledR}$ and Botox $^{\circledR}$, using equivalent doses in 300 patients, with 256 patients completing the study. There was no difference in efficacy or adverse effects between the two formulations. ${ }^{7}$

Conclusions. For patients with blepharospasm, BoNT injection is probably effective with minimal side effects (two Class II studies). After dosage adjustment, Botox ${ }^{\circledR}$ and $\mathrm{Xeomin}^{\circledR}$ are probably equivalent (one Class I study), and Botox $\left.^{(}\right)$and Dysport ${ }^{\circledR}$ are possibly equivalent (one Class II and one Class III study).
Recommendation. BoNT injection should be considered as a treatment option for blepharospasm (Level B).

Clinical context. The evidence supporting BoNT use in blepharospasm is suboptimal. The large magnitude of benefits in the initial open label studies and the lack of other effective therapy likely have discouraged efforts to study BoNT in larger and more properly controlled clinical trials.

Hemifacial spasm. Hemifacial spasm is characterized by a combination of unilateral clonic and tonic spasms of the muscles innervated by the facial nerve. Treatment options include oral pharmacologic therapies, including carbamazepine, baclofen, and benzodiazepine, and resulting in limited efficacy, and microvascular decompression of the facial nerve, a highly invasive procedure. Encompassed in the category of VII nerve disorders, hemifacial spasm is FDA approved. One efficacy trial of BoNT fulfilled criteria for Class II, and one for Class III (table e-2). The Class II study ${ }^{8}$ of 11 patients was a prospective, blinded trial with four arms: an arbitrary dose based on clinical experience of between 2.5 and 10 units of BoNT-A (Botox ${ }^{\circledR}$ ), half the dose, double the dose, and saline placebo. Each subject cycled through the four treatment arms in a random order. Using a clinical scale to rate videotapes and a patient subjective scale, $84 \%$ had objective improvement with at least one of the active doses with a trend for better response with higher dose; only one patient improved on placebo. Seventy-nine percent reported subjective benefit lasting a mean of 2.8 months with active therapy. Weakness of the face, generally mild, was the most common adverse effect (97\%). Other adverse effects included bruising, diplopia, ptosis, and headache.

A Class III study ${ }^{9}$ was a double-blind, prospective, parallel design study with only four patients per group using individualized therapy (dose range 2.5 to 40 units) with BoNT-A (Botox ${ }^{\circledR}$ ) in the active arm. Ninety-three patients studied in an open label fashion were also reported. There was greater improvement on a clinical scale with BoNT than with a saline placebo. Benefit lasted a mean of 3.8 months. Side effects, present in $63 \%$ of patients, were generally mild and included dry eye, mouth droop, and ptosis.

One Class II study compared Botox ${ }^{\circledR}$ and Dysport $^{\circledR}$ in a parallel design without placebo control or blinded raters. ${ }^{6}$ There was a dose ratio of $4: 1$ for Dysport ${ }^{\circledR}$ to Botox. ${ }^{\circledR}$ The primary endpoint (duration of action) and other endpoints (number of booster doses needed, latency of effect, clinical efficacy, and frequency of adverse reactions) were 
similar for the two products. Benefit lasted 2.63.0 months.

Conclusions. BoNT is possibly effective with minimal side effects for the treatment of hemifacial spasm (one Class II and one Class III study). Botox $^{\circledR}$ and Dysport ${ }^{\circledR}$, after dosage adjustment, are possibly equivalent in efficacy (one Class II study).

Recommendation. BoNT injection may be considered as a treatment option for hemifacial spasm (Level C).

Clinical context. The evidence supporting BoNT use in hemifacial spasm is suboptimal. The large magnitude of effects in the initial open label studies likely has discouraged efforts to study BoNT in properly controlled clinical trials. No studies have compared BoNT with the other major treatment alternatives, including oral pharmacologic and surgical therapy.

Cervical dystonia. Cervical dystonia (CD) is a focal dystonia causing involuntary activation of the muscles of the neck and shoulders resulting in abnormal, sustained, and painful posturing of the head, neck, and shoulders. There are limited data assessing oral medications for cervical dystonia. Recent surgical studies, including deep brain stimulation, show promise. Out of approximately 80 studies of BoNT in the treatment of CD, 14 controlled studies were identified, including seven Class I studies (four with BoNT-A, three with BoNT-B) (table e-3). ${ }^{10-16}$ Botox $^{\circledR}$ and Myobloc ${ }^{\circledR}$ are FDA approved for use in CD.

Three Class I studies enrolled BoNT-naïve CD subjects. One study ${ }^{16}$ evaluated 55 subjects over 12 weeks. Subjects were stratified by primary type of torticollis and randomized to BoNT-A or placebo. Maximal benefit occurred at 6 weeks following injection with improvement in functional capacity, head turning, pain, and subjective assessment. Adverse events included dysphagia, neck weakness, and fatigue.

A second Class I study compared low, intermediate, and high doses of BoNT-A to placebo in 75 BoNT-naive CD subjects with rotational torticollis. ${ }^{12}$ At 1 month, the intermediate- and high-dose groups demonstrated improvement compared to placebo $(p<0.05)$. Subjective improvement occurred in $45-50 \%$ and was significant at 1 and 2 months for the high-dose group, and at 2 months for the intermediate-dose group. More adverse events occurred with higher doses, including neck weakness, voice changes, and dysphagia.

A third Class I study in BoNT-naïve subjects compared BoNT-A and trihexyphenidyl (mean dose $16.25 \mathrm{mg}$; range $4-24 \mathrm{mg}$ ) at 3 months following treatment. ${ }^{15}$ BoNT-A was superior to tri- hexyphenidyl for TWSTRS disability (2 points), Tsui scale (5 points), and general health perception (6 points). Although there was greater improvement in TWSTRS pain score with BoNT (2 points), this did not reach statistical significance. The total TWSTRS and TWSTRS severity scores were not given for either group. The trihexyphenidyl group had more adverse events $(76$ events vs 31 for BoNT-A, $p<0.0001$ ).

Four Class I studies enrolled subjects with previous response to BoNT. Three studies assessed safety and efficacy of BoNT-B, ${ }^{10,11,14}$ and one assessed safety and efficacy of BoNT-A. ${ }^{13}$ One of these studies randomized 109 subjects with CD to placebo, medium dose, or high dose of BoNT-B. ${ }^{10}$ There was an improvement in total TWSTRS scores at 1 month for both treatment groups compared to placebo (medium dose $p=0.01$; high dose $p=0.01$, with return to baseline by 3 months. The TWSTRS severity and pain subscales and patient and physician global scales showed similar benefit. Adverse events were greater in the BoNT-B treated groups, with dry mouth and pain occurring in a ose-dependent way. All adverse events were mild. similar study assessed the effect of BoNT-B ompared to placebo in 77 patients with $\mathrm{CD}$ who developed resistance to BoNT-A. ${ }^{11}$ At 1 month following injection, the BoNT-B group had more improvement in total TWSTRS score $(21 \%$ vs $4 \%$ in placebo, $p=0.0001$ ). Treatment with BoNT-B improved the TWSTRS severity, disability, and pain subscales, and physician and patient global scores. Dry mouth occurred in $3 \%$ of placebo subjects and $44 \%$ of the BoNT-B group. A study evaluated BoNT-A compared to placebo in 80 subjects with CD previously treated with BoNTA. ${ }^{13}$ This study showed improvement in TWSTRS total score and each of the subscales of the TWSTRS for severity, disability, and pain at 1 month with only blurred vision and neck weakness occurring more frequently than placebo.

Conclusion. BoNT is established as safe and effective for the treatment of CD (seven Class I studies).

\section{Recommendations}

- BoNT injection should be offered as a treatment option to patients with cervical dystonia (Level A).

- BoNT is probably more efficacious and better tolerated in patients with CD than treatment with trihexyphenidyl (Level B).

Clinical context. BoNT has longstanding and widespread use in the treatment of $\mathrm{CD}$, a condition without effective alternative medical therapies. There are no data to compare BoNT with surgical 
treatment of CD. The role of electromyography has not been established for cervical dystonia.

Focal limb dystonia. Most studies of BoNT in focal limb dystonia deal with the upper extremity. Although no controlled trials of BoNT exist for lower limb dystonia, some larger studies do include these patients. The term "focal hand dystonia" is used here to encompass writer's cramp, other occupational hand dystonia, and nontaskspecific hand dystonia. The pattern of limb dystonia varies widely among patients. There are no effective alternative medical or well-established surgical therapies for focal limb dystonia. The use of BoNT to treat limb dystonia requires thoughtful technique including customization of doses and muscle selection.

There is Class I and Class II evidence for focal limb dystonia ${ }^{17}$ (table e-4). The Class I trial randomized 40 patients with writer's cramp in a double-blind design to BoNT or an equivalent volume of saline placebo. Injected muscles were chosen based on clinical examination. Participants with inadequate or no response were offered a second injection 1 month later. The primary outcome measure was the subject's stated desire to continue injection Seventy percent of those randomized to BoNT wished to continue treatment compared to $32 \%$ of those receiving placebo $(p=0.03)$. Significant improvement was also found in BoNT-injected subjects compared to those receiving placebo in secondary outcome measuresincluding a visual analog scale, symptoms severity scale, writer's cramp rating scale, and assessment of writing speed, but not in the functional status scale. Temporary weakness and pain at the injection site were the only adverse events reported.

One Class II trial ${ }^{18}$ was a prospective, doubleblind, crossover study of 17 patients with several forms of limb dystonia, including lower extremity (3 patients) and secondary dystonia (4 patients). Subjects received a series of four injections in random order, one with a dose of BoNT that the investigators judged to be "optimal," one at half the optimal dose, one at double the dose, and one with saline placebo. Using a patient subjective scale, $82 \%$ of patients receiving BoNT had benefit compared to $6 \%$ (one patient) who received placebo. Using physician rating of videotapes, $59 \%$ improved with active treatment and 38\% with placebo (not significant). There was no dose-response relationship for benefit, and there was a large degree of interobserver variability. The authors attributed the lack of significance in physician ratings to an inadequate outcome eval- uation. The main side effect was focal weakness that followed $53 \%$ of BoNT injections and was more likely at the higher doses. Weakness occurred with $13 \%$ of placebo injections. Other adverse effects included muscle stiffness, pain, and malaise.

Another Class II study used a placebocontrolled, double-blind, crossover design in 20 patients with writer's cramp. ${ }^{19}$ Muscle selection was guided by clinical examination; dose of BoNT-A was based on investigator experience. Outcome assessments included evaluation of writing speed, accuracy, writing samples, and patients' subjective report. There was significant improvement with BoNT therapy in the objective measures, but not in patients' own assessments. Focal weakness was the only adverse effect and was severe enough to worsen pen control in one patient. The authors noted that this study evaluated only the first active treatment session that a patient received, so that the benefit obtained was likely not optimal.

Another Class II trial was a double-blind, placebo-controlled, crossover in 10 patients with focal hand dystonia. ${ }^{20}$ Muscles and BoNT-A doses yere selected and optimized during a period of open treatment preceding the controlled study. Patient subjective rating and observer rating of videotapes during activities applicable to individual dystonia were the outcome measures. Eight patients had improved subjective rating and six had improved videotape rating with BoNT compared with placebo. Weakness was present in the injected muscles in $80 \%$ of subjects with active treatment.

Three Class II studies evaluated technical issues of BoNT administration (table e-4). In one study, a blinded, randomized, crossover design was used to compare continuous muscle activation to immobilization immediately after BoNT injection. $^{21}$ Blinded evaluation of handgrip strength and writing revealed a significant increase in focal weakness with continuous muscle activity, but no subjective or objective improvement in writing. In another Class II study, patients were randomized to one of two muscle localization techniques: EMG recording or electrical stimulation. ${ }^{22}$ Injections guided by either technique were equally effective in producing weakness in the target muscle. The accuracy of muscle localization with and without EMG was evaluated in a third trial. ${ }^{23}$ Only $37 \%$ of needle placements based on surface anatomy were localized in the targeted muscle.

Conclusions. BoNT is probably effective for the treatment of focal upper extremity limb dystonia 
(one Class I and three Class II studies). While a few patients in one Class II study suggest that BoNT may be effective for lower extremity dystonia, the data are inadequate to provide a recommendation.

Recommendation. BoNT should be considered as a treatment option for focal upper extremity dystonia (Level B).

Clinical context. The treatment of focal limb dystonia with BoNT presents challenges, particularly in achieving sufficient neuromuscular blockade to alleviate dystonic movements without causing excessive muscle weakness. While many clinicians advocate EMG or nerve stimulation guidance to optimize needle localization for injection, further data are needed to establish this recommendation.

Laryngeal dystonia. Laryngeal dystonia (spasmodic dysphonia) generally presents as adductor type (ADSD) and less frequently as abductor type of spasmodic dysphonia (ABSD). ADSD is characterized by a "strain-strangle" voice, while ABSD produces a breathy and hypophonic voice. There are no effective alternative medical or surgical therapies for spasmodic dysphonia. There is one Class I study of BoNT $^{24}(\mathrm{n}=13)$ of patients with ADSD. This double-blind, randomized, parallel group study compared seven patients receiving BoNT with six receiving saline. Outcome measures included instrumental quantitative measures of voice function and patient ratings. Significant benefit was found in the BoNT-injected group $(p=0.01)$ (table e-5). One Class III study found that the addition of voice therapy following BoNT in ADSD prolonged benefit from BoNT treatment.25 Another found that voice rest 30 minutes after BoNT injection prolonged the benefit of BoNT. ${ }^{26}$ One Class III study of 15 patients with $\mathrm{ABSD}^{27}$ did not find a significant difference using either percutaneous or endoscopic injection technique.

Conclusions. BoNT is probably effective for the treatment of ADSD (one Class I study). There is insufficient evidence to support a conclusion of effectiveness for BoNT in ABSD.

\section{Recommendations}

- BoNT should be considered as a treatment option for adductor spasmodic dysphonia (Level B).

- There is insufficient evidence to support or refute the use of BoNT in abductor spasmodic dysphonia (Level U).

Clinical context. The evidence supporting BoNT use in laryngeal disorders is suboptimal. While most clinicians utilize EMG targeting for laryngeal injections, the utility of this technique is not established in comparative trials. Dramatic re- sults in the initial open label studies and the lack of other effective therapy likely have discouraged efforts to study BoNT in larger and more properly controlled clinical trials.

Tics. Typically associated with Tourette syndrome, tics are relatively brief, intermittent movements (motor tics) or sounds (vocal or phonic tics), usually preceded by a premonitory sensation. ${ }^{28}$ While antidopaminergic drugs (neuroleptics) are often effective in treating troublesome multifocal tics, these drugs often produce undesirable side effects, particularly in patients with focal tics, such as blinking, blepharospasm, head jerking, neck twisting, and loud vocalizations, including coprolalia. Although confined to a limited anatomic distribution, such focal tics may be a source of embarrassment and may result in functional blindness, local discomfort, and social isolation.

In initial open label Class IV studies, injections of BoNT in the muscles involved in the motor and phonic tics was associated with a moderate to marked reduction in the intensity and frequency of the tics, and nearly complete abolishment of the premonitory sensation. In a Class IV study of 35 patients treated in 115 sessions for troublesome or disabling tics, the mean peak effect response was 2.8 (range $0=$ no effect; $4=$ marked improvement in both severity and function). ${ }^{29}$ The mean duration of benefit was 3.4 months (up to 10.5). Latency to onset of benefit was 3.8 days (up to 10). Twenty-one of 25 patients $(84 \%)$ with notable premonitory sensory symptoms derived marked relief of these symptoms from BoNT (mean benefit: $70.6 \%$ ).

In a Class II study of 18 patients with simple motor tics, there was a $39 \%$ reduction in the number of tics per minute within 2 weeks after injection with BoNT, as compared to a $6 \%$ increase in the placebo group ( $p=0.004$, table e-6). ${ }^{30}$ In addition, there was a 0.46 reduction in "urge scores" with BoNT compared to a 0.49 increase in the placebo group ( $p=0.02$ ). This study lacked the power to show significant differences in other measured variables, such as severity score, tic suppression, pain, and patient global impression. The full effect of BoNT may not have been appreciated at 2 weeks. The study employed a single treatment session protocol that does not reflect the clinical practice of evaluating patients after several adjustments in doses and sites of injections. Furthermore, since subjects "did not rate themselves as significantly compromised by their treated tics," it is likely that their symptoms were relatively mild at baseline.

Conclusions. BoNT is possibly effective for the treatment of motor tics (one Class II study). 
There are insufficient data to determine the effectiveness of BoNT in phonic tics (one Class IV study).

Recommendation. BoNT may be considered as a treatment option for motor tics (Level C).

Clinical context. There are no data to compare the efficacy of BoNT and neuroleptics in the treatment of tic disorders.

Tremor. Tremor, an oscillatory movement produced by alternating or synchronous contractions of antagonistic muscles, is the most common movement disorder. While propranolol and primidone usually ameliorate mild or moderate essential tremor, pharmacotherapy is usually not sufficient to control a high-amplitude tremor that impairs activities of daily living. In such cases of disabling tremor, local injection of BoNT may be used before considering more aggressive intervention such as thalamic deep brain stimulation.

A Class II placebo-controlled study evaluated 25 patients with hand tremor of 2 (moderate) to 4 (severe) on the tremor severity rating scale ${ }^{31}$ (table e-7). Subjects were randomized to receive either 50 units of BoNT-A $\left(\right.$ Botox $^{\circledR}$ ) or placebo injections into the wrist flexors and extensors of dominant limb. If patients failed to respond to the initial injection, they were eligible to receive another injection of 100 units 4 weeks later.

Rest, postural, and kinetic tremor were evaluated at 2- to 4-week intervals over a 16-week study period, using tremor severity rating scales, accelerometry, and assessments of improvement and disability. There was significant improvement on the tremor severity rating scale 4 weeks after injection in patients treated with BoNT as compared to placebo, and this effect was maintained for the duration of the study. Four weeks after injection, $75 \%$ of BoNT-treated patients vs $27 \%$ of placebo-treated patients $(p<0.05)$ reported mild to moderate improvement. Functional rating scales did not improve although trends were observed for some items. Postural accelerometry measurements showed a $30 \%$ reduction in amplitude in 9 of 12 BoNT-treated subjects and in 1 of 9 placebo-treated subjects $(p<0.05)$. Although all patients treated with BoNT reported some degree of finger weakness, no severe, irreversible, or unexpected adverse events occurred.

There were similar results in another Class II multicenter, double-blind, controlled trial that utilized a similar protocol and involved 133 patients with essential tremor. ${ }^{32}$ The patients were randomized to receive 50 or $100 \mathrm{U}$ of Botox ${ }^{\circledR}$ into wrist flexors and extensors and were followed for 4 months. The study showed significant improvement in pos- tural tremor, but only minimal improvement in kinetic tremor and functional assessments.

The study design of both Class II studies limits their applicability to clinical practice. Both used a rigid treatment protocol that employed a fixed BoNT dose and a predetermined set of muscles. In practice, dosages and injected muscles are often individually chosen on the basis of tremor pattern.

An underpowered Class II study of 10 patients with head tremor ${ }^{33}$ did not show a statistically significant benefit in BoNT-treated patients. There are two Class IV open-label studies in voice tremor $^{34,35}$ that showed modest improvement from baseline in objective acoustic and subjective measures after unilateral or bilateral BoNT injection.

Conclusions. BoNT injection of forearm muscles is probably effective in reducing the tremor amplitude in patients with essential hand tremor (two Class II studies). The benefits must be considered in conjunction with the common adverse effect of muscle weakness associated with BoNT injection. Existing data are insufficient to draw a conclusion on the use of BoNT in the treatment of head and voice tremor.

Recommendation. BoNT should be considered as treatment option for essential hand tremor in those patients who fail treatment with oral agents (Level B).

Clinical context. Oral agents and deep brain stimulation are alternative treatments for essential tremor. There are presently no data comparing the efficacy of BoNT to these treatment modalities. By reducing or eliminating BoNT injection into wrist extensors, the complications of finger and hand weakness may be reduced. However, no controlled data employing the new methodology are available.

Summary. The evidence supporting the use of BoNT in movement disorders is summarized in the table.

\section{RECOMMENDATIONS FOR FUTURE RESEARCH}

- Many of the recommendations for future research provided in the companion article on BoNT for spasticity are also pertinent to movement disorders. Additional recommendations follow.

- Further placebo-controlled trials are needed to evaluate the efficacy and safety of BoNT for several movement disorders, particularly blepharospasm, hemifacial spasm, lower limb dystonia, phonic tics, and head and voice tremor.

- To the extent that issues of feasibility and ethics make such studies unlikely, other 
Table Summary table for botulinum toxin in the treatment of movement disorders

\begin{tabular}{|c|c|c|c|c|c|c|c|}
\hline Disorder & Class & $\begin{array}{l}\text { No. of } \\
\text { subjects }\end{array}$ & Outcome measures & Adverse events & Conclusions & Recommendations ${ }^{*}$ & Limitations \\
\hline Blepharospasm & 2 Class II & 17 & Subjective, Fahn scale & $\begin{array}{l}\text { Dry eye, tearing, } \\
\text { ptosis, diplopia, } \\
\text { lid edema, } \\
\text { ecchymosis }\end{array}$ & Probably effective & $B$ & $\begin{array}{l}\text { Lack of controlled studies } \\
\text { due to dramatic efficacy }\end{array}$ \\
\hline Hemifacial spasm & $\begin{array}{l}1 \text { Class II } \\
\text { and } 1 \\
\text { Class III }\end{array}$ & 19 & $\begin{array}{l}\text { Clinical, video, } \\
\text { subjective }\end{array}$ & $\begin{array}{l}\text { Weakness, } \\
\text { bruising, } \\
\text { diplopia, ptosis, } \\
\text { dry eye }\end{array}$ & Possibly effective & $\mathrm{C}$ & $\begin{array}{l}\text { Lack of controlled studies } \\
\text { due to dramatic efficacy; } \\
\text { surgery major alternate Rx }\end{array}$ \\
\hline Cervical dystonia & 7 Class I & 584 & TWSTRS, Tsui, disability & $\begin{array}{l}\text { Dysphagia, neck } \\
\text { weakness, dry } \\
\text { mouth, pain }\end{array}$ & $\begin{array}{l}\text { Established safe and } \\
\text { effective }\end{array}$ & $A$ & No effective alternate $R x$ \\
\hline $\begin{array}{l}\text { Focal limb } \\
\text { dystonia (UE) }\end{array}$ & 3 Class II & 47 & $\begin{array}{l}\text { Video, handwriting } \\
\text { accuracy/speed }\end{array}$ & $\begin{array}{l}\text { Focal weakness, } \\
\text { pain }\end{array}$ & Probably effective & B & No effective alternate Rx \\
\hline $\begin{array}{l}\text { Focal limb } \\
\text { dystonia (LE) }\end{array}$ & 1 Class II & 3 & Video, subjective & $\begin{array}{l}\text { Focal weakness, } \\
\text { pain }\end{array}$ & Data inadequate & None & No effective alternate Rx \\
\hline $\begin{array}{l}\text { Laryngeal } \\
\text { dystonia }\end{array}$ & 1 Class I & 13 & $\begin{array}{l}\text { Quant voice } f x \text {, patient } \\
\text { ratings }\end{array}$ & $\begin{array}{l}\text { Breathiness, } \\
\text { bleeding }\end{array}$ & $\begin{array}{l}\text { Adductor SD: probably } \\
\text { effective; abductor SD: } \\
\text { inadequate data }\end{array}$ & $B$ & No effective alternate $R x$ \\
\hline Motor tics & 1 Class II & 18 & $\begin{array}{l}\text { Tic frequency, urge } \\
\text { scores, global disability }\end{array}$ & Focal weakness & Possibly effective & $\mathrm{C}$ & $\begin{array}{l}\text { No comparative data with } \\
\text { oral agents }\end{array}$ \\
\hline $\begin{array}{l}\text { Essential tremor } \\
\text { (UE) }\end{array}$ & 2 Class II & 158 & $\begin{array}{l}\text { Tremor rating, } \\
\text { subjective (MD/pt); SIP }\end{array}$ & Focal weakness & Probably effectiv & & $\begin{array}{l}\text { No comparative data with } \\
\text { oral agents }\end{array}$ \\
\hline
\end{tabular}

${ }^{*}$ Classification of recommendations is available on the Neurology ${ }^{\circledR}$ Web site at www.neurology

$A=$ Should be offered; $B$ = should be considered; $C$ = may be considered; $U E$ = upper ex dysphonia; MD = physicion; $\mathrm{pt}=$ patient; SIP = Sickness Impact Profile.

strategies should be considered to proye the Brashear has received speaker honoraria from Allergan, Solstice, and efficacy of these treatments in the current era of evidence-based medicine.

DISCLAIMER This statement is provided as an educational service of the American Academy of Neurology. It is based on an assessment of current scientific and clinical information. It is not intended to include all possible proper methods of care for a particular neurologic problem or all legitimate criteria for choosing to use a specific procedure. Neither is it intended to exclude any reasonable alternative methodologies. The AAN recognizes that specific patient care decisions are the prerogative of the patient and the physician caring for the patient, based on all of the circumstances involved. The clinical context section is made available in order to place the evidencebased guideline(s) into perspective with current practice habits and challenges. No formal practice recommendations should be inferred.

\section{ACKNOWLEDGMENT}

The authors thank Dr. Mitchell Brin for his contributions in the early phases of this project.

\section{DISCLOSURE}

The authors report the following conflicts: Dr. Simpson has received speaker honoraria and research support from Allergan, Merz, and Solstice, Inc., and performs botulinum toxin injections. Dr. Blitzer has received speaker honoraria from Allergan, Solstice, and Merz; research support from Allergan; and performs botulinum toxin injections. Dr.

forms botulinum toxin injections and has received payment for expert testimony. Dr. Comella has received speaker honoraria from Jazz Pharmaceutical, Merz Pharmaceutical, and UCB Pharmaceutical; research support from Allergan, Dystonia Study Group, and Solstice; and performs botulinum toxin injections. Dr. Dubinsky has received speaker honoraria from Allergan and research support from Allergan, MERZINC, and Solstice Neurosciences. Dr. Dubinsky holds financial interest in Abbott Laboratories (spouse), performs botulinum toxin injections, and presents annual courses at AANEM on chemodenervation. Dr. Hallett holds financial interest in Amylin Pharmaceuticals, Eli Lilly, Genetech, Genzyme, Healthsouth Corp., Medtronic, Pfizer, St. Jude Medical, Triad Hospitals, United Healthcare, and Valeant Pharmaceuticals International and performs botulinum toxin injections. Dr. Jankovic has received speaker honoraria from Allergan and Merz Pharmaceutical, research support from Allergan, Ipsen, and Merz Pharmaceutical, and performs botulinum toxin injections. Dr. Karp performs botulinum toxin injections. Dr. Ludlow holds financial interest in Fidelity Biotechnology (family member). Dr. Miyasaki has received research support from Boehringer Ingelheim, Huntington Study Group, NIH, Solvay, Solstice, and Teva. Dr. Naumann has received speaker honoraria from Ipsen and Allergan and performs botulinum toxin injections. Dr. So holds financial interest in Satoris Inc., and has received research support from NIH, Pfizer, Inc., and NeurogesX, Inc.

\section{REFERENCES}

1. Simpson DM, Gracies J-M, Graham HK, et al. Assessment: Botulinum neurotoxin for the treatment of spasticity (an evidence-based review): Report of the Therapeutics and Technology Assessment Subcommittee of the American Academy of Neurology. Neurology 2008;70:1707-1714.

2. Naumann M, So Y, Argoff CE, et al. Assessment: Botulinum neurotoxin in the treatment of autonomic disorders and pain (an evidence-based review): Report of the Therapeutics and Technology Assessment Subcom- 
mittee of the American Academy of Neurology. Neurology 2008;70:1699-1706.

3. Girlanda P, Quartarone A, Sinicropi S, Nicolosi C, Messina C. Unilateral injection of botulinum toxin in blepharospasm: single fiber electromyography and blink reflex study. Mov Disord 1996;11:27-31.

4. Jankovic J, Orman J. Botulinum A toxin for cranialcervical dystonia: a double-blind, placebo-controlled study. Neurology 1987;37:616-623.

5. Nussgens Z, Roggenkamper P. Comparison of two botulinum-toxin preparations in the treatment of essential blepharospasm. Graefes Arch Clin Exp Ophthalmol 1997;235:197-199.

6. Sampaio C, Ferreira JJ, Simoes F, et al. DYSBOT: a single-blind, randomized parallel study to determine whether any differences can be detected in the efficacy and tolerability of two formulations of botulinum toxin type A-Dysport and Botox-assuming a ratio of 4:1. Mov Disord 1997;12:1013-1018.

7. Roggenkamper P, Jost WH, Bihari K, Comes G, Grafe S. Efficacy and safety of a new botulinum toxin type A free of complexing proteins in the treatment of blepharospasm. J Neural Transm 2006;113:303-312.

8. Yoshimura DM, Aminoff MJ, Tami TA, Scott AB. Treatment of hemifacial spasm with botulinum toxin. Muscle Nerve 1992;15:1045-1049.

9. Park YC, Lim JK, Lee DK, Yi SD. Botulinum a toxi treatment of hemifacial spasm and blepharospasm. J Korean Med Sci 1993;8:334-340.

10. Brashear A, Lew MF, Dykstra DD, et al. Safety and efficacy of NeuroBloc (botulinum toxin type B) in type A-responsive cervical dystonia. Neurology 1999;53: 1439-1446.

11. Brin MF, Lew MF, Adler CH, et al. Safety and effieacy of NeuroBloc (botulinum toxin type B) in type A-resistant cervical dystonia. Neurology 1999;53:1431-1438.

12. Poewe W, Deuschl G, Nebe A, et al, What is the optimal dose of botulinum toxin $\mathrm{A}$ in the treatment of cervical dystonia? Results of a double blind, placebo controlled, dose ranging study using Dysport. German Dystonia Study Group. JNeurol Neurosurg Psychiatry 1998;64:13-17.

13. Truong D, Duane DD, Jankovic J, et al. Efficacy and safety of botulinum type A toxin (Dysport) in cervical dystonia: results of the first US randomized, double-blind, placebo-controlled study. Mov Disord 2005;20:783-791.

14. Lew MF, Adornato BT, Duane DD, et al. Botulinum toxin type B: a double-blind, placebo-controlled, safety and efficacy study in cervical dystonia. Neurology 1997;49:701-707.

15. Brans JW, Lindeboom R, Snoek JW, et al. Botulinum toxin versus trihexyphenidyl in cervical dystonia: a prospective, randomized, double-blind controlled trial. Neurology 1996;46:1066-1072.

16. Greene P, Kang U, Fahn S, Brin M, Moskowitz C, Flaster E. Double-blind, placebo-controlled trial of botulinum toxin injections for the treatment of spasmodic torticollis. Neurology 1990;40:1213-1218.

17. Kruisdijk JJ, Koelman JH, Ongerboer de Visser BW, de Haan RJ, Speelman JD. Botulinum toxin for writer's cramp: a randomised, placebo-controlled trial and 1-year follow-up. J Neurol Neurosurg Psychiatry 2007; 78:264-270.

18. Yoshimura DM, Aminoff MJ, Olney RK. Botulinum toxin therapy for limb dystonias. Neurology 1992;42: 627-630.

19. Tsui JK, Bhatt M, Calne S, Calne DB. Botulinum toxin in the treatment of writer's cramp: a double-blind study. Neurology 1993;43:183-185.

20. Cole R, Hallett M, Cohen LG. Double-blind trial of botulinum toxin for treatment of focal hand dystonia. Mov Disord 1995;10:466-471.

21. Chen R, Karp BI, Goldstein SR, Bara-Jimenez W, Yaseen Z, Hallett M. Effect of muscle activity immediately after botulinum toxin injection for writer's cramp. Mov Disord 1999;14:307-312.

22. Geenen C, Consky E, Ashby P. Localizing muscles for botulinum toxin treatment of focal hand dystonia. Can J Neurol Sci 1996;23:194-197.

23. Molloy FM, Shill HA, Kaelin-Lang A, Karp BI. Accuracy of muscle localization without EMG: implications for treatment of limb dystonia. Neurology 2002;58:805-807.

24. Troung DD, Rontal M, Rolnick M, Aronson AE, Mistura K. Double-blind controlled study of botulinum toxin in adductor spasmodic dysphonia. Laryngoscope 1991;101:630-634.

25. Murry T, Woodson GE. Combined-modality treatment of adductor spasmodic dysphonia with botulinum toxin and voice therapy. J Voice 1995;9:460-465.

26. Wong DL, Adams SG, Irish JC, Durkin LC, Hunt EJ, Charlton MP. Effect of neuromuscular activity on the response to botulinum toxin injections in spasmodic dysphonia. J Otolaryngol 1995;24:209-216.

27. Bielamowicz S, Squire S, Bidus K, Ludlow CL. Assessment of posterior cricoarytenoid botulinum toxin injections in patients with abductor spasmodic dysphonia. Ann Otol Rhinol Laryngol 2001;110:406-412.

28. Jankovic J. Tourette's syndrome. N Engl J Med 2001; 345:1184-1192.

29. Kwak CH, Hanna PA, Jankovic J. Botulinum toxin in the treatment of tics. Arch Neurol 2000;57:1190-1193.

30. Marras C, Andrews D, Sime E, Lang AE. Botulinum toxin for simple motor tics: a randomized, double-blind, controlled clinical trial. Neurology 2001;56:605-610.

31. Jankovic J, Schwartz K, Clemence W, Aswad A, Mordaunt J. A randomized, double-blind, placebocontrolled study to evaluate botulinum toxin type A in essential hand tremor. Mov Disord 1996;11:250-256.

32. Brin MF, Lyons KE, Doucette J, et al. A randomized, double masked, controlled trial of botulinum toxin type A in essential hand tremor. Neurology 2001;56:1523-1528.

33. Pahwa R, Busenbark K, Swanson-Hyland EF, et al. Botulinum toxin treatment of essential head tremor. Neurology 1995;45:822-824.

34. Warrick P, Dromey C, Irish JC, Durkin L, Pakiam A, Lang A. Botulinum toxin for essential tremor of the voice with multiple anatomical sites of tremor: a crossover design study of unilateral versus bilateral injection. Laryngoscope 2000;110:1366-1374.

35. Adler $\mathrm{CH}$, Bansberg SF, Hentz JG, et al. Botulinum toxin type A for treating voice tremor. Arch Neurol 2004;61:1416-1420. 


\title{
Neurology
}

\begin{abstract}
Assessment: Botulinum neurotoxin for the treatment of movement disorders (an evidence-based review) [RETIRED]: Report of the Therapeutics and Technology Assessment Subcommittee of the American Academy of Neurology

D. M. Simpson, A. Blitzer, A. Brashear, et al. Neurology 2008;70;1699-1706

DOI 10.1212/01.wnl.0000311389.26145.95
\end{abstract}

This information is current as of May 5, 2008

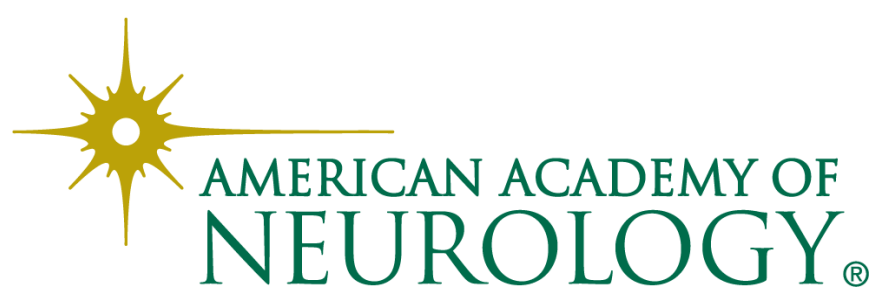




\section{Updated Information \& Services}

\section{Supplementary Material}

\section{References}

Citations

Subspecialty Collections

\section{Permissions \& Licensing}

\section{Reprints}

including high resolution figures, can be found at: http://n.neurology.org/content/70/19/1699.full

Supplementary material can be found at: http://n.neurology.org/content/suppl/2009/11/06/70.19.1699.DC2 http://n.neurology.org/content/supp1/2008/05/29/70.19.1699.DC1

This article cites 35 articles, 16 of which you can access for free at: http://n.neurology.org/content/70/19/1699.full\#ref-list-1

This article has been cited by 14 HighWire-hosted articles: http://n.neurology.org/content/70/19/1699.full\#\#otherarticles

This article, along with others on similar topics, appears in the following collection(s):

\section{All Movement Disorders}

http://n.neurology.org/cgi/collection/all_movement_disorders Blepharospasm

http://n.neurology.org/cgi/collection/blepharospasm

Botulinum toxin

http://n.neurology.org/cgi/collection/botulinum_toxin

Clinical trials Systematic review/meta analysis

http://n.neurology.org/cgi/collection/clinical_trials_systematic_review_ meta_analysis_

\section{Dystonia}

http://n.neurology.org/cgi/collection/dystonia

Information about reproducing this article in parts (figures,tables) or in its entirety can be found online at:

http://www.neurology.org/about/about_the_journal\#permissions

Information about ordering reprints can be found online: http://n.neurology.org/subscribers/advertise

Neurology ${ }^{\circledR}$ is the official journal of the American Academy of Neurology. Published continuously since 1951, it is now a weekly with 48 issues per year. Copyright . All rights reserved. Print ISSN: 0028-3878. Online ISSN: 1526-632X.

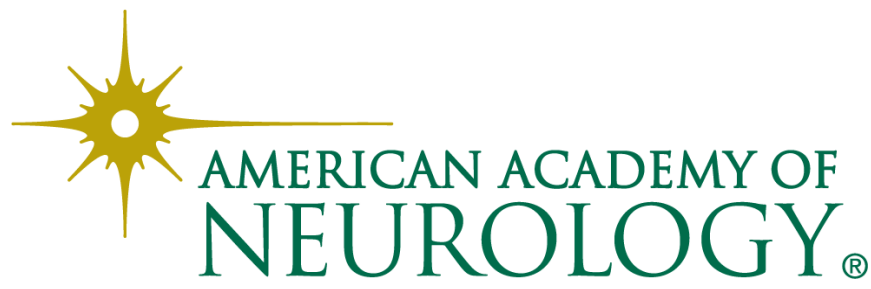

